\title{
Anästhesiologie im Wandel?
}

G entleman, this is no humbug“ - Mit diesen Worten kommentierte der berühmte Chirurg John Collins Warren an der Harvard Universität in Boston (Massachusetts, USA) die erste erfolgreiche Äthernarkose, die der Zahnarzt William Thomas Green Morten am 16.10.1846 durchführte. Er leitete damit eine Revolution in der operativen Medizin ein. Die weitere Entwicklung und Einführung anästhesiologischer Verfahren in die klinische Medizin ermöglichte nun ausgedehnte und invasive sowie zeitlich nahezu unbegrenzte Eingriffe, die heute Routine in vielen Krankenhäusern sind.

Die moderne Anästhesie hat in Deutschland eine eher kurze Geschichte. Im Jahr 2003 feierte die Deutsche Gesellschaft für Anästhesiologie und Intensivmedizin (DGAI) ihren 50. Geburtstag. Damit ist sie eine der jüngeren Fachgesellschaften Europas. Man kann allerdings in Europa lange nach einer Bündelung derartig vielseitiger und interessanter Strukturen und Aufgaben suchen, wie sie die deutsche Anästhesiologie auszeichnen. Der verspätete Eintritt der deutschen Anästhesie in das Spektrum der großen klinischen Fächer, überwiegend Folge der Isolation der deutschen Medizin bis 1945, brachte aber auch große Chancen mit sich. Bereiche wie die sich entwickelnde Notfall- und Schmerzmedizin - von der Intensivmedizin ganz zu schweigen - lagen quasi zur „Inbesitznahme" auf der Straße. Die Pioniere des Faches nutzten diese Chance, sodass Anästhesisten heute in den erwähnten Fachbereichen der Medizin über ihre Tätigkeit als „Narkosearzt“ hinaus eine herausragende Rolle spielen.

Die Weiterbildungsordnung der Bundesärztekammer ordnet dem Fachgebiet die Allgemein-, Regionalund Lokalanästhesie einschließlich deren Vor- und Nachbereitung, die Aufrechterhaltung der vitalen Funktionen während operativer und diagnostischer Eingriffe sowie intensivmedizinische, notfallmedizinische und schmerztherapeutische Maßnahmen zu. Sie beschreibt also im Wesentlichen die vorgenannten Tätigkeitsfelder des Anästhesisten, wenngleich diese Aufgaben immer im interdisziplinären Zusammenhang zu sehen sind. Dabei ist die Kooperation mit allen anderen klinischen Disziplinen ein Gebot und die Voraussetzung für die unbestreitbaren Erfolge in Lehre, Forschung und Krankenversorgung.
In den nachfolgenden Beiträgen des aktuellen klinikarzt-Schwerpunkthefts kommen prominente Vertreter der vier Säulen der Anästhesiologie zu Wort, die ansprechen, was bislang in den einschlägigen Publikationen wenig Platz fand: Zum Beispiel fordern die Autoren die weitere Qualifizierung des anästhesiologischen Hilfspersonals. In den USA, aber auch in mehreren europäischen Ländern wie Skandinavien, der Schweiz, Holland und teilweise auch in Frankreich sind die Anästhesieschwestern und -pfleger inzwischen wesentlich spezifischer ausgebildet als in Deutschland. Dementsprechend übernehmen sie dort auch qualifiziertere Aufgaben - bis hin zu einfachen Anästhesien, allerdings unter der Aufsicht eines Facharztes. Angesichts des zunehmenden Personalmangels in der Anästhesie scheint auch bei uns eine Diskussion unvermeidlich, wie unter Aufsicht der Anästhesisten bestimmte Ausbildungsgänge für das Anästhesieassistenzpersonal besser den spezifischen Anforderungen in der Anästhesiologie und der Intensivmedizin angepasst werden können, ohne dabei die ärztliche Verantwortung für die Anästhesie infrage zu stellen.

Ähnlich, wenn auch nicht völlig vergleichbar, ist die Situation in der Notfallmedizin. Hier ließ sich durch die zunehmende Qualifizierung von Rettungsassistenten längst ein großer Fortschritt erzielen. Offensichtlich kann eine frühzeitige Intervention, wie zum Beispiel die Frühdefibrillation durch den Rettungsassistenten, das Outcome von Patienten mit Kammerflimmern erheblich verbessern.

Mit diesem kurzen Einblick in das Themenspektrum des vorliegenden Heftes wünsche ich Ihnen viel Spaß beim Lesen der verschiedenen Fassetten der modernen Anästhesiologie.

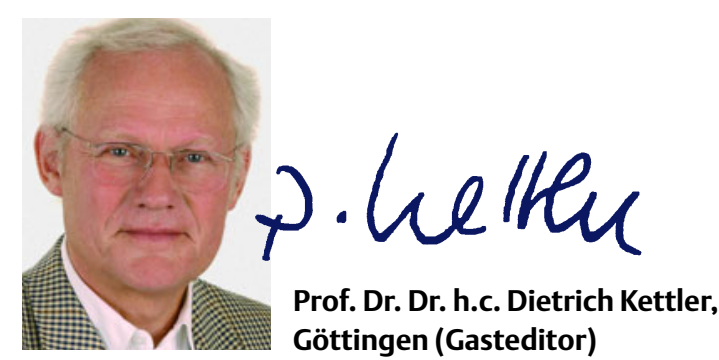

